\title{
Multistable Systems with Hidden and Self-Excited Scroll Attractors Generated via Piecewise Linear Systems
}

\author{
R. J. Escalante-González and Eric Campos \\ Division of Applied Mathematics, Institute for Scientific and Technological Research of San Luis Potosí, \\ Camino a la Presa San José 2055, Lomas 4 Sección 78216, San Luis Potosí, Mexico
}

Correspondence should be addressed to Eric Campos; eric.campos@ipicyt.edu.mx

Received 4 January 2020; Revised 26 February 2020; Accepted 23 March 2020; Published 14 April 2020

Guest Editor: Sundarapandian Vaidyanathan

Copyright (c) 2020 R. J. Escalante-González and Eric Campos. This is an open access article distributed under the Creative Commons Attribution License, which permits unrestricted use, distribution, and reproduction in any medium, provided the original work is properly cited.

\begin{abstract}
In this work, we present an approach to design a multistable system with one-directional (1D), two-directional (2D), and threedirectional (3D) hidden multiscroll attractor by defining a vector field on $\mathbb{R}^{3}$ with an even number of equilibria. The design of multistable systems with hidden attractors remains a challenging task. Current design approaches are not as flexible as those that focus on self-excited attractors. To facilitate a design of hidden multiscroll attractors, we propose an approach that is based on the existence of self-excited double-scroll attractors and switching surfaces whose relationship with the local manifolds associated to the equilibria lead to the appearance of the hidden attractor. The multistable systems produced by the approach could be explored for potential applications in cryptography, since the number of attractors can be increased by design in multiple directions while preserving the hidden attractor allowing a bigger key space.
\end{abstract}

\section{Introduction}

Piecewise linear systems that display scroll attractors have been studied since the publication of the well-known Chua's circuit. The attractor exhibited by Chua's circuit is an example of chaotic attractor whose chaotic nature has been explained through the Shilnikov method. Some works have extended this system in order to obtain a greater number of scrolls or different geometries. According to [1], an attractor with three or more scrolls in the attractor is considered a multiscroll attractor. Recently in [2], the generation of scroll attractors via multistable systems have been observed.

According to [3], there are two classes of attractors, one of them is a class called self-excited attractors that includes all the attractors excited by unstable equilibria, i.e., the basin of attraction intersects with an arbitrarily small open neighborhood of equilibria [4]. Examples of this class are the well-known Lorenz attractor [5] and the scroll attractor of Chua's circuit [6]. The other class is called hidden attractors and their basins of attractions do not contain neighborhoods of equilibria. Some hidden attractors have been studied in
[7-19]. There are some works focused on control systems with hidden attractors, as in references [20, 21]. Most works related to multiscroll attractors are based on the first class. Multiscroll attractors have been reported in $[12,13,18,19,22-26]$. In [27], a system with a multiscroll chaotic sea was introduced.

Multistability can be considered undesirable for some applications, so some works focus on how to avoid this behavior. For example, in [28], a method that allows to transform a periodic or chaotic multistable system into a monostable was studied, and some experiments were carried out with a fiber laser doped with erbium. However, for some applications it may be considered desirable to be able to switch from monostable to bistable behavior, for example, in [29], a parameterized method to design multivibrator circuits with stable, monostable, and bistable regimes was proposed.

Some works deal with multistable systems with infinite number of equilibria and their electronic realization $[30,31]$.

A study on the widening of the basin of attraction of a class of piecewise linear (PWL) systems was recently 
performed in [2]. In this work, a bifurcation from a bistable system with two self-excited double-scroll attractors to a multistable system with two self-excited attractors and one hidden attractor was reported. Other study on the emergence of hidden double-scroll attractors in a class of PWL systems is reported in [32].

Based on the observations made in previous works, a question of whether or not it is possible to generate a hidden multiscroll attractor with scrolls along more than one direction emerges. Depending on the number of directions in which the scrolls in the attractor extend, they are usually referred to as one-directional (1D), two-directional (2D), and three-directional (3D) grid scroll attractors.

Here, we introduce an approach for the construction of multistable PWL systems that exhibit hidden multiscroll attractors with $1 \mathrm{D}, 2 \mathrm{D}$, and $3 \mathrm{D}$ grid arrangements. In Section 2, a system with a chaotic double-scroll self-excited attractor is introduced. In Section 3, the construction is extended to $1 \mathrm{D}$ grid scroll self-excited attractor; then, the equilibria are separated by pairs to generate multistable systems with hidden and self-excited attractors. In Section 4, the construction is generalized to $1 \mathrm{D}$ grid scroll hidden attractor. In Section 5, the construction is generalized to 2D and 3D grid scroll hidden attractors; in Section 6, conclusions are given.

\section{Heteroclinic Chaos}

Let $P=\left\{P_{1}, \ldots, P_{\eta}\right\}(\eta>1)$ be a finite partition of $X \subset \mathbb{R}^{3}$, that is, $X=\cup_{1 \leq i \leq \eta} P_{i}$, and $P_{i} \cap P_{j}=\varnothing$ for $i \neq j$. The approach to generate hidden attractors is based on the existence of self-excited attractors; thus, the class of systems considered in this work are those that present a saddle equilibrium point in each element of the partition $P$ that is called an atom.

We denote the closure of a set $P_{i}$ as $c l\left(P_{i}\right)$. For each pair of adjacent atoms $P_{i}$ and $P_{j}, i \neq j, S W_{i, j}=\operatorname{cl}\left(P_{i}\right) \cap \operatorname{cl}\left(P_{j}\right)$ is the switching surface.

Consider a dynamical system $T: X \longrightarrow X$ whose dynamic is given by

$$
\dot{\mathbf{x}}=A \mathbf{x}+f(\mathbf{x}) B,
$$

where $\mathbf{x}=\left(x_{1}, x_{2}, x_{3}\right)^{T} \in \mathbb{R}^{3}$ is the state vector and $A=\left\{\alpha_{i j}\right\} \in \mathbb{R}^{3 \times 3}$ is a linear operator whose matrix is as follows:

$$
A=\left(\begin{array}{ccc}
\frac{a}{3}+\frac{2 c}{3} & b & \frac{2 c}{3}-\frac{2 a}{3} \\
-\frac{b}{3} & a & \frac{2 b}{3} \\
\frac{c}{3}-\frac{a}{3} & -b & \frac{2 a}{3}+\frac{c}{3}
\end{array}\right),
$$

where $a, b \in \mathbb{R}^{+}$, and $c \in \mathbb{R}^{-}$. Thus, the linear operator $A$ has a negative real eigenvalue $\lambda_{1}=c$ with the corresponding eigenvector $v_{1}$ and a pair of complex conjugate eigenvalues with positive real part, $\lambda_{2}=a+i b$ and $\lambda_{3}=a-i b$, with the corresponding eigenvectors $v_{2}$ and $v_{3}$, respectively. The eigenvectors are given by

$$
\begin{aligned}
& v_{1}=\left(\begin{array}{l}
1 \\
0 \\
1 \\
\frac{1}{2}
\end{array}\right), \\
& v_{2}=\left(\begin{array}{c}
0 \\
-1 \\
0
\end{array}\right), \\
& v_{3}=\left(\begin{array}{c}
-1 \\
0 \\
1
\end{array}\right) .
\end{aligned}
$$

$B=\left(\beta_{1}, \beta_{2}, \beta_{3}\right)^{T} \in \mathbb{R}^{3}$ is a constant vector, and $f: X \longrightarrow \mathbb{R}$ is a functional such that $f(\mathbf{x}) B$ is a constant vector in each atom $P_{i}$, and there exists an equilibrium point $\mathbf{x}_{e q_{i}}^{*}=\left(x_{1_{e q_{i}}^{*}}^{*}, x_{2_{e q_{i}}^{*}}^{*} x_{3_{e q_{i}}^{*}}^{*}\right)^{T}=-f(\mathbf{x}) A^{-1} B$, with $i=1, \ldots, \eta$, in each atom $P_{i}$. Thus, in each atom $P_{i}$ there exists a saddle equilibrium point with a local stable manifold of dimension one given by $W_{\mathbf{x}_{e q_{i}}^{*}}^{s}=\left\{\mathbf{x}+\mathbf{x}_{e q_{i}}^{*}: \mathbf{x} \in \operatorname{span}\left\{v_{1}\right\}\right\}$. A two-dimensional local unstable manifold is given by $W_{\mathbf{x}_{e q_{i}}^{*}}^{u}=$ $\left\{\mathbf{x}+\mathbf{x}_{e q_{i}}^{*}: \mathbf{x} \in \operatorname{span}\left\{v_{2}, v_{3}\right\}\right\}$.

We begin to explain the generation of chaotic attractors by first considering a partition with two atoms $P=\left\{P_{1}, P_{2}\right\}$ and the constant vector $B \in \mathbb{R}^{3}$ given by

$$
B=\left(\begin{array}{c}
-\frac{a}{3}-\frac{2 c}{3} \\
\frac{b}{3} \\
\frac{a}{3}-\frac{c}{3}
\end{array}\right),
$$

and the functional $f$ given by

$$
f(\mathbf{x})= \begin{cases}-\alpha, & \mathbf{x} \in P_{1}, \\ \alpha, & \mathbf{x} \in P_{2},\end{cases}
$$

with $0<\alpha \in \mathbb{R}$.

Please note that the vector $-B$ is the first column of the linear operator $A$, and thus, system (1) can be rewritten as $\dot{\mathbf{x}}=A\left(x_{1}-f(\mathbf{x}), x_{2}, x_{3}\right)^{T}$. Then, the functional $f(\mathbf{x})$ determines the location of the equilibria along the $x_{1}$-axis. So, $\mathbf{x}_{e q_{i}}^{*}=\left(x_{1_{e q_{i}}}^{*}, x_{2_{e q_{i}}^{*}}^{*}, x_{3_{e q_{i}}^{*}}^{*}\right)^{T} \in P_{i}, i=1,2$.

Proposition 1. If the PWL system (1) is given by (2), (4), and (5), then the functional $f(\mathbf{x})$ determines the location of the equilibria along the $x_{1}$-axis. 
Proof. Let $A=\left[A_{1}, A_{2}, A_{3}\right]$ be the linear operator so that each $A_{i}, i=1,2,3$, is a column vector. Since $B=-A_{1}$, then $\dot{\mathbf{x}}=A \mathbf{x}+f(\mathbf{x}) B$ can be rewritten as

$$
\dot{\mathbf{x}}=\left(x_{1}-f(\mathbf{x})\right) A_{1}+x_{2} A_{2}+x_{3} A_{3},
$$

and then

$$
\dot{x}=A\left(x_{1}-f(\mathbf{x}), x_{2}, x_{3}\right)^{T} .
$$

Now, in order to find the equilibria, we equate the vector field to zero:

$$
\mathbf{0}=A\left(x_{1}-f(\mathbf{x}), x_{2}, x_{3}\right)^{T} .
$$

Since $A \neq 0$, it follows that

$$
\mathbf{x}_{e q}^{*}=(f(\mathbf{x}), 0,0)^{T} .
$$

Thus, the equilibria is determined by $f(\mathbf{x})$ given by (5) along the $x_{1}$-axis.

According to $f(\mathbf{x})$, the first components of the equilibria fulfill that $x_{1}^{*}<x_{1}^{*}$.

The switching plane $S W$ has associated an equation $\widehat{A} x_{1}+\widehat{B} x_{2}+\widehat{C} x_{3}+D=\mathbf{N} \cdot \mathbf{x}^{\mathrm{T}}+D=0$, with $\widehat{A}>0$, and $\mathbf{N}=(\widehat{A}, \widehat{B}, \widehat{C})$ is the normal vector. Then, the atoms $P_{i}$, $i=1,2$, are defined as follows:

$$
\begin{aligned}
& P_{1}=\left\{\mathbf{x} \in \mathbb{R}^{3}: x_{3}>0, \quad \mathbf{N} \cdot \mathbf{x}^{\mathbf{T}} \leq-D\right\} \cup\left\{\mathbf{x} \in \mathbb{R}^{3}: x_{3} \leq 0, \quad \mathbf{N} \cdot \mathbf{x}^{\mathbf{T}}<-D\right\}, \\
& P_{2}=\left\{\mathbf{x} \in \mathbb{R}^{3}: x_{3}>0, \quad \mathbf{N} \cdot \mathbf{x}^{\mathbf{T}}>-D\right\} \cup\left\{\mathbf{x} \in \mathbb{R}^{3}: x_{3} \leq 0, \quad \mathbf{N} \cdot \mathbf{x}^{\mathbf{T}} \geq-D\right\} \text {. }
\end{aligned}
$$

Assumption 1. The switching plane $S W$ intersects the $x_{1}$-axis at the midpoint between the equilibrium points $\mathbf{x}_{e q_{1}}^{*}$ and $\mathbf{x}_{e q_{2}}^{*}$.

Proposition 2. Under Assumption 1, each atom $P_{i}$ given by (10), $i=1,2$, contains an equilibrium point of the $P W L$ system (1) given by (2), (4), and (5).

Proof. We want to prove that if a system is given by (1), (2), (4), and (5), then $\exists \mathbf{x}_{e q_{1}}^{*} \in P_{1}$ and $\exists \mathbf{x}_{e q_{2}}^{*} \in P_{2}$. From (9) and (5), the two equilibrium points are

$$
\mathbf{x}_{e q_{1}}^{*}=(-\alpha, 0,0)^{T} \text { and } \mathbf{x}_{e q_{2}}^{*}=(\alpha, 0,0)^{T} .
$$

From Assumption 1, the parameter $D$ can be defined as $D=-x_{s w_{1}} \widehat{A}$, where $x_{s w_{1}} \in\left(x_{1_{e q_{1}}}^{*}, x_{1_{e q_{2}}^{*}}^{*}\right)$ is the intersection of $S W$ with the $x_{1}$-axis:

$$
\begin{aligned}
& \mathbf{N} \cdot \mathbf{x}_{e q_{1}}^{*}=-\alpha \widehat{A}, \\
& \mathbf{N} \cdot \mathbf{x}_{e q_{2}}^{*}=\alpha \widehat{A} .
\end{aligned}
$$

Thus,

$$
\begin{aligned}
& \mathbf{N} \cdot \mathbf{x}_{e q_{1}}^{*}<-D, \\
& \mathbf{N} \cdot \mathbf{x}_{e q_{2}}^{*}>-D,
\end{aligned}
$$

from $(10) \mathbf{x}_{e q_{1}}^{*} \in P_{1}$ and $\mathbf{x}_{e q_{2}}^{*} \in P_{2}$.

Proposition 3. If $S W=\operatorname{cl}\left(P_{1}\right) \cap \operatorname{cl}\left(P_{2}\right)=\left\{\mathbf{x} \in \mathbb{R}^{3}: 2 x_{1}-\right.$ $\left.x_{3}=0\right\}$, the stable and unstable manifolds of the PWL system (1) given by (2), (4), and (5) intersect at two points given by $\mathbf{x}_{i n_{1}}=((\alpha / 3), 0,(2 \alpha / 3))^{T}$ and $\mathbf{x}_{i n_{2}}=(-(\alpha / 3), 0,-(2 \alpha / 3))^{T}$.

Proof. The equilibrium points are located at $\mathbf{x}_{e q_{1}}^{*}=(-\alpha, 0,0)^{T}$ and $\mathbf{x}_{e q_{2}}^{*}=(\alpha, 0,0)^{T}$. In each atom $P_{i}$, the equilibrium point $\mathbf{x}_{e q_{i}}^{*}$ has a local stable manifold (14) of dimension one given by $W_{\mathbf{x}_{e q_{i}}^{*}}^{s}=\left\{\mathbf{x}+\mathbf{x}_{e q_{i}}^{*}: \mathbf{x} \in \operatorname{span}\left\{v_{1}\right\}\right\}$ and a two-dimensional local ${ }^{e q_{i}}$ unstable manifold given by
$W_{\mathbf{x}_{e q_{i}}^{*}}^{u}=\left\{\mathbf{x}+\mathbf{x}_{e q_{i}}^{*}: \mathbf{x} \in \operatorname{span}\left\{v_{2}, v_{3}\right\}\right\}$. Thus, the local manifolds are given as follows:

$$
\begin{aligned}
W_{\mathbf{x}_{e q_{1}}^{*}}^{s} & =\left\{\mathbf{x} \in P_{1}: x_{1}+\alpha=2 x_{3}, x_{2}=0\right\}, \\
W_{\mathbf{x}_{e q_{1}}^{*}}^{u} & =\left\{\mathbf{x} \in P_{1}: x_{1}+x_{3}=-\alpha\right\}, \\
W_{\mathbf{x}_{e q_{2}}^{*}}^{s} & =\left\{\mathbf{x} \in P_{2}: x_{1}-\alpha=2 x_{3}, \quad x_{2}=0\right\}, \\
W_{\mathbf{x}_{e q_{2}}^{*}}^{u} & =\left\{\mathbf{x} \in P_{2}: x_{1}+x_{3}=\alpha\right\} .
\end{aligned}
$$

According to (18), the stable and unstable manifolds, and the intersection points are given by

$$
\begin{aligned}
& \mathbf{x}_{i n_{1}}=c l\left(W_{\mathbf{x}_{e q_{1}}^{*}}^{s}\right) \cap c l\left(W_{\mathbf{x}_{e q_{2}}^{*}}^{u}\right)=((\alpha / 3), 0,(2 \alpha / 3))^{T}, \\
& \mathbf{x}_{i n_{2}}=c l\left(W_{\mathbf{x}_{e q_{2}}^{*}}^{s}\right) \cap c l\left(W_{\mathbf{x}_{e q_{1}}^{*}}^{u}\right)=(-(\alpha / 3), 0,(2 \alpha / 3))^{T} .
\end{aligned}
$$

These points belong to $S W$.

Assumption 2. The parameters $a$ and $b$ control the oscillation around the equilibrium point $\mathbf{x}_{e q_{i}}^{*}$, and we consider $b / a>10$.

Proposition 4. The hyperbolic system given by (1), (2), (4), and (5) generates a pair of heteroclinic orbits if the switching surface between the atoms $P_{1}$ and $P_{2}$ is given by the plane $S W=\operatorname{cl}\left(P_{1}\right) \cap \operatorname{cl}\left(P_{2}\right)=\left\{\mathbf{x} \in \mathbb{R}^{3}: 2 x_{1}-x_{3}=0\right\}$.

Proof. We want to show that there exist initial conditions $\mathbf{x}_{01}, \mathbf{x}_{02} \in S W$, such that two solution curves $\varphi\left(\mathbf{x}_{01}, t\right)$ and $\varphi\left(\mathbf{x}_{02}, t\right)$ of the hyperbolic system given by (1), (2), (4), and (5) fulfill that $\varphi\left(\mathbf{x}_{01}, t\right) \longrightarrow \mathbf{x}_{e q_{1}}^{*}$ and $\varphi\left(\mathbf{x}_{02}, t\right) \longrightarrow \mathbf{x}_{e q_{2}}^{*}$ as $t \longrightarrow \infty$ and $\varphi\left(\mathbf{x}_{01}, t\right) \longrightarrow \mathbf{x}_{e q_{2}}^{*}$ and $\varphi\left(\mathbf{x}_{02}, t\right) \longrightarrow \mathbf{x}_{e q_{1}}^{*}$ as $t \longrightarrow-\infty$; in particular, these initial conditions correspond to the intersection points $c l\left(W_{\mathbf{x}_{e q_{1}}^{*}}^{s}\right) \cap c l\left(W_{\mathbf{x}_{e q_{2}}^{*}}\right)$ and $c l\left(W_{\mathbf{x}_{e q_{2}}^{*}}^{s}\right) \cap c l\left(W_{\mathbf{x}_{e_{1}}^{*}}^{u}\right)$. 
From (2), the linear operator $A$ can be expressed as

$$
A=Q E Q^{-1}
$$

where $Q=\left[\begin{array}{lll}v_{1} & v_{2} & v_{3}\end{array}\right]$ and

$$
E=\left(\begin{array}{ccc}
c & 0 & 0 \\
0 & a & -b \\
0 & b & a
\end{array}\right) .
$$

The intersection points $\mathbf{x}_{i n_{1}}$ and $\mathbf{x}_{i n_{2}}$ belong to $S W$ and $\mathbf{x}_{i n_{1}} \in P_{1}$ and $\mathbf{x}_{i n_{2}}=P_{2}$. Because these points $\mathbf{x}_{i n_{1}}$ and $\mathbf{x}_{i n_{2}}$ belong to the stable manifolds $W_{\mathbf{x}_{e q_{1}}^{*}}^{s}$ and $W_{\mathbf{x}_{e q_{2}}^{*}}^{s}$, respectively, they are points whose trajectories remain in atoms $P_{1}$ and $P_{2}$, respectively.

By definition, $\mathbf{x}_{1_{\text {eq }}}^{*}=-\mathbf{x}_{1_{\text {eq }}}^{*}$; then, the $x_{2}$-axis belongs to the plane $S W$. The sets $c l\left(W_{\mathbf{x}_{e q_{i}}^{*}}^{u}\right) \cap S W$, for $i=1,2$, can be written as follows:

$$
\left\{(0, \epsilon, 0)^{T}+\mathbf{x}_{i n_{i}}: \in \in \mathbb{R}\right\}, \text { for } i=1,2 .
$$

Consider the following changes of coordinates $\mathbf{z}^{(i)}=Q^{-1}\left(\mathbf{x}-\mathbf{x}_{e q_{i}}^{*}\right)$, for $i=1,2$. Then, the vector field in $\mathbf{z}^{(i)}$ coordinates for the space given by the atom $P_{i}$ is given by $\dot{z}^{(i)}=E \mathbf{z}^{(i)}$, with $i=1,2$.

Since $Q^{-1}(0, \epsilon, 0)^{T}=(0,-\epsilon, 0)^{T}$, the sets given by $(22)$ in $\mathbf{z}^{(i)}$ coordinates are given as follows:

$$
\left\{(0, \epsilon, 0)^{T}+Q^{-1}\left(\mathbf{x}_{i n_{i}}-\mathbf{x}_{e q_{i}}^{*}\right): \epsilon \in \mathbb{R}\right\}, \quad \text { for } \quad i=1,2 \text {, }
$$

where $\mathbf{z}_{i n_{i}}^{(i)}=Q^{-1}\left(\mathbf{x}_{i n_{i}}-\mathbf{x}_{e q_{i}}^{*}\right)=\left((-1)^{i+1} 4 \alpha / 3,0,0\right)^{T}$ is a point on the $z_{1}^{(i)}$-axis that corresponds to the transformation of the intersection points $\mathbf{x}_{i n_{i}} \in P_{i}$ to $\mathbf{z}_{i n_{i}}^{(i)} \in\left\{Q^{-1}\left(\mathbf{x}-\mathbf{x}_{e q_{i}}^{*}\right): \mathbf{x} \in P_{i}\right\}$, for $i=1,2$.

When $t>0, \varphi\left(\mathbf{x}_{i n_{1}}, t\right)$ remains in the atom $P_{1}$, the transformation of $\mathbf{x}_{i n_{1}}$ under $Q^{-1}\left(\mathbf{x}_{i n_{1}}-\mathbf{x}_{e q_{1}}^{*}\right)$ is $\mathbf{z}_{i n_{1}}^{(1)}=(4 \alpha / 3,0,0)^{T}$. In a similar way, when $t>0, \varphi\left(\mathbf{x}_{i n_{2}}, t\right)$, remains in the atom $P_{2}$, the transformation of $\mathbf{x}_{i n_{2}}$ under $Q^{-1}\left(\mathbf{x}_{i n_{2}}-\mathbf{x}_{e q_{2}}^{*}\right)$ is $\mathbf{z}_{i n_{2}}^{(2)}=(-4 \alpha / 3,0,0)^{T}$. So, $\mathbf{z}_{i n_{i}}^{(i)}$ belongs to the stable manifold $W_{\mathbf{z}_{e q}^{*}}^{s}$, for $i=1,2$; then, the trajectories $\mathbf{z}^{(i)}(t)=e^{E t} \mathbf{z}_{i n_{i}}^{(i)} \longrightarrow 0$ when $t \longrightarrow \infty$. This implies that

$$
\begin{aligned}
& \lim _{t \longrightarrow \infty} \varphi\left(\mathbf{x}_{i n_{1}}, t\right)=\mathbf{x}_{e q_{1}}^{*}, \\
& \lim _{t \longrightarrow \infty} \varphi\left(\mathbf{x}_{i n_{2}}, t\right)=\mathbf{x}_{e q_{2}}^{*} .
\end{aligned}
$$

When $t<0, \varphi\left(\mathbf{x}_{i n_{1}}, t\right)$ leaves the atom $P_{1}$ and enters to atom $P_{2}$, the transformation of $\mathbf{x}_{i n_{1}}$ under $Q^{-1}\left(\mathbf{x}_{i n_{1}}-\mathbf{x}_{e q}^{*}\right)$ to $\mathbf{z}_{i n_{1}}^{(2)} \in\left\{Q^{-1}\left(\mathbf{x}-\mathbf{x}_{e q_{2}}^{*}\right) n: \mathbf{x} \in \operatorname{cl}\left(P_{2}\right)^{\prime}\right\}$ is $\mathbf{z}_{i n_{1}}^{(2)}=(0,0,2 \alpha / 3)^{f}$. In a similar way, when $t<0, \varphi\left(\mathbf{x}_{i n_{2}}, t\right)$ leaves the atom $P_{2}$ and enters to atom $P_{1}$, the transformation of $\mathbf{x}_{i n_{2}}$ under $Q^{-1}\left(\mathbf{x}_{i n_{2}}-\mathbf{x}_{e q_{1}}^{*}\right)$ to $\mathbf{z}_{i n_{2}}^{(1)} \in\left\{Q_{(j)}^{-1}\left(\mathbf{x}-\mathbf{x}_{e q_{1}}^{*}\right): \mathbf{x} \in \operatorname{cl}\left(P_{1}\right)\right\}$ is $\mathbf{z}_{i n_{2}}^{(1)}=(0,0,-2 \alpha / 3)^{T}$. Thus, $\mathbf{z}_{i n_{i}}^{(j)}=\left(0,0,(-1)^{j} 2 \alpha / 3\right)^{T}$ is a point on the axis $z_{3}^{(j)}$ and belongs to $c l\left(W_{\mathbf{z}_{e q_{j}}^{*}}^{u}\right)$ for $i, j \in\{1,2\}$ and $i \neq j$.
With the uncoupled system in $\mathbf{z}^{(i)}$ coordinates, we can analyze the flow on the plane $z_{2}^{(i)}-z_{3}^{(i)}$ and see how the flow converges at the equilibrium point $\mathbf{z}_{e q_{j}}^{*(i)}$ when $t \longrightarrow-\infty$ :

$$
\begin{aligned}
& \dot{z}_{2}^{(i)}=a z_{2}^{(i)}-b z_{3}^{(i)}, \\
& \dot{z}_{3}^{(i)}=b z_{2}^{(i)}+a z_{3}^{(i)}, \\
& z_{2}^{(i)} \dot{z}_{2}^{(i)}+z_{3}^{(i)} \dot{z}_{3}^{(i)}=a\left(\left(z_{2}^{(i)}\right)^{2}+\left(z_{3}^{(i)}\right)^{2}\right) . \\
& \text { If } r^{2}=\left(z_{2}^{(i)}\right)^{2}+\left(z_{3}^{(i)}\right)^{2}, \text { then } r \dot{r}=a r^{2} \\
& \dot{r}=a r, \\
& r=r_{0} e^{a t .}
\end{aligned}
$$

As $0<a \in \mathbb{R}$, so $r \longrightarrow 0$ when $t \longrightarrow-\infty$. Then, the trajectories $\mathbf{z}^{(i)}(t)=e^{E t} \mathbf{z}_{\text {in }_{i}}^{(i)} \longrightarrow 0$ when $t \longrightarrow-\infty$. This implies that

$$
\lim _{t \longrightarrow-\infty} \varphi\left(\mathbf{x}_{i n_{1}}, t\right)=\mathbf{x}_{e q_{2}}^{*}, \quad \text { and } \quad \lim _{t \longrightarrow-\infty} \varphi\left(\mathbf{x}_{i n_{2}}, t\right)=\mathbf{x}_{e q_{1}}^{*},
$$

Thus, the heteroclinic orbits are defined as

$$
\begin{aligned}
& H O_{1}=\left\{\mathbf{x} \in \varphi\left(\mathbf{x}_{i n_{1}}, t\right): t \in(-\infty, \infty)\right\}, \\
& H O_{2}=\left\{\mathbf{x} \in \varphi\left(\mathbf{x}_{{i n_{2}}_{2}}, t\right): t \in(-\infty, \infty)\right\} .
\end{aligned}
$$

As example, consider systems (1), (2), (4), and (5) with the parameters $a=0.2, b=5, c=-3, \alpha=1$, which fulfills (10) with the switching surface $S W=\operatorname{cl}\left(P_{1}\right) \cap \operatorname{cl}\left(P_{2}\right)$ $=\left\{\mathbf{x} \in \mathbb{R}^{3}: 2 x_{1}-x_{3}=0\right\}$. Then, heteroclinic chaos emerges from this system, a double-scroll attractor is exhibited, as it is shown in Figure 1(b), for the initial condition $\mathbf{x}_{0}=(0,0,0)^{T}$.

\section{Multiple Self-Excited Attractors}

A study on the widening of the basins of attraction of multistable switching dynamical system with symmetrical equilibria is performed in [2]. In this study, the increment of distance between the equilibria of the self-excited doublescroll attractors increases the basin of attraction of both attractors. The study also reveals that, for the system under study, there is a distance at which a hidden double-scroll attractor emerges.

Based on the idea of generating hidden scroll attractors from sufficiently separated self-excited attractors, we consider now a partition with more atoms $P=\left\{P_{1}, P_{2}, P_{3}, P_{4}\right\}$ along with the PWL system (1), with $A$ and $B$ given by (2) and (4), respectively. For this partition, the functional $f(\mathbf{x})$ is defined in the four atoms as follows:

$$
f(\mathbf{x})= \begin{cases}-\alpha-\gamma, & \mathbf{x} \in P_{1}, \\ \alpha-\gamma, & \mathbf{x} \in P_{2}, \\ -\alpha+\gamma, & \mathbf{x} \in P_{3}, \\ \alpha+\gamma, & \mathbf{x} \in P_{4},\end{cases}
$$




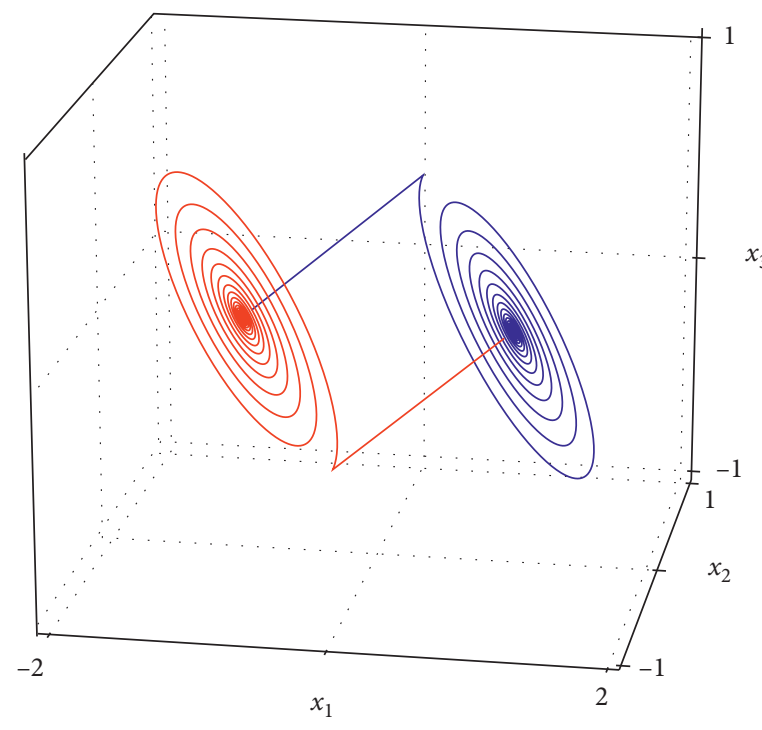

(a)

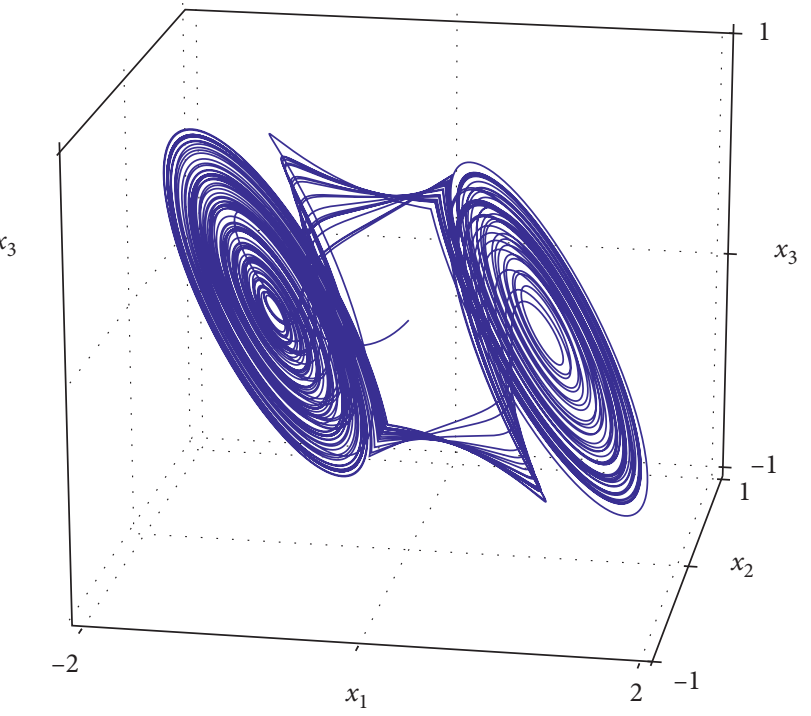

(b)

FIgURE 1: In (a) the heteroclinic loop of system (1), (2), (4), and (5) with the switching surface $\left\{x \in \mathbb{R}^{3}: 2 x_{1}-x_{3}=0\right\}$ and the parameters $a=0.2, b=5, c=-3, \alpha=1$ and in (b) a double-scroll attractor that emerges from an heteroclinic loop using the following initial condition $\mathbf{x}_{0}=(0,0,0)^{T}$.

where $0<\alpha, \gamma \in \mathbb{R}$ and $\gamma>\alpha$. Now, there are four atoms and each atom contains an equilibrium point, $\mathbf{x}_{e q_{i}}^{*}=\left(x_{1_{e q_{i}}^{*}}^{*}\right.$, $\left.x_{2_{e q q_{i}}}^{*}, x_{3_{e q_{i}}}^{*}\right)^{T} \in P_{i}, i=1,2,3,4$. The first components of the equilibrium points fulfill that $x_{1_{e_{i}}}^{*}<x_{1_{e_{q_{i+1}}}}^{*}, i=1,2,3$.

There are three switching planes and each switching plane $S W_{i, i+1}$ crosses the $x_{1}$-axis and is located between the equilibrium points $\mathbf{x}_{e q_{i}}^{*}$ and $\mathbf{x}_{e q_{i+1}}^{*}, i=1,2,3$.

The switching plane $S W_{i j}$ with $i, j \in\{1, \ldots, \eta\}$ has associated an equation $A_{i j} x_{1}+B_{i j} x_{2}+C_{i j} x_{3}+D_{i j}=\mathbf{N}_{i j}$. $\mathbf{x}^{T}+D_{i j}=0$, where $A_{i j}>0$ and $\mathbf{N}_{\mathbf{i j}}=\left(A_{i j}, B_{i j}, C_{i j}\right)$ is the normal vector. Then, in order to know if a point $\mathbf{x}$ belongs to a $P_{i}$ the following conditions are considered:

$$
\begin{aligned}
& \text { If } \quad x \in\left\{\mathbf{x} \in \mathbb{R}^{3}: x_{3}>0, \mathbf{N}_{i j} \cdot \mathbf{x}^{T} \leq-D_{i j}\right\}, \\
& \text { then } \quad x \in P_{k} \text { for a } k \leq i, \\
& \text { If } x \in\left\{\mathbf{x} \in \mathbb{R}^{3}: x_{3}>0, \mathbf{N}_{i j} \cdot \mathbf{x}^{T}>-D_{i j}\right\}, \\
& \text { then } \quad x \in P_{k} \text { for a } k \geq j, \\
& \text { If } x \in\left\{\mathbf{x} \in \mathbb{R}^{3}: x_{3} \leq 0, \mathbf{N}_{i j} \cdot \mathbf{x}^{T} \geq-D_{i j}\right\}, \\
& \text { then } \quad x \in P_{k} \text { for a } k \geq j, \\
& \text { If } x \in\left\{\mathbf{x} \in \mathbb{R}^{3}: x_{3} \leq 0, \mathbf{N}_{i j} \cdot \mathbf{x}^{T}<-D_{i j}\right\}, \\
& \text { then } \quad x \in P_{k} \text { for a } k \leq i .
\end{aligned}
$$

If $\mathbf{x} \in P_{k}$ for a $k \geq i$ and $\mathbf{x} \in P_{k}$ for a $k \leq i$, it follows that $\mathbf{x} \in P_{i}$.
The equilibria are located on the $x_{1}$-axis at

$$
\begin{aligned}
& \mathbf{x}_{e q_{1}}^{*}=\left[\begin{array}{c}
-(\gamma+\alpha) \\
0 \\
0
\end{array}\right], \\
& \mathbf{x}_{e q_{2}}^{*}=\left[\begin{array}{c}
-(\gamma-\alpha) \\
0 \\
0
\end{array}\right], \\
& \mathbf{x}_{e q_{3}}^{*}=\left[\begin{array}{c}
(\gamma-\alpha) \\
0 \\
0
\end{array}\right], \\
& \mathbf{x}_{e q_{4}}^{*}=\left[\begin{array}{c}
(\gamma+\alpha) \\
0 \\
0
\end{array}\right],
\end{aligned}
$$

so $\mathbf{x}_{e q_{1}}^{*} \in P_{1}, \mathbf{x}_{e q_{2}}^{*} \in P_{2}, \mathbf{x}_{e q_{3}}^{*} \in P_{3}$, and $\mathbf{x}_{e q_{4}}^{*} \in P_{4}$.

Assumption 3. The distance between the self-excited attractors should be big enough to allow the existence of a hidden double-scroll attractor, so we consider $(\gamma / \alpha) \geq 10$.

Consider three switching surfaces with the following orientation:

$$
\begin{aligned}
& S W_{1,2}=\operatorname{cl}\left(P_{1}\right) \cap \operatorname{cl}\left(P_{2}\right)=\left\{\mathbf{x} \in \mathbb{R}^{3}: 2 x_{1}-x_{3}=-2 \gamma\right\}, \\
& S W_{2,3}=\operatorname{cl}\left(P_{2}\right) \cap \operatorname{cl}\left(P_{3}\right)=\left\{\mathbf{x} \in \mathbb{R}^{3}: 2 x_{1}-x_{3}=0\right\}, \\
& S W_{3,4}=\operatorname{cl}\left(P_{3}\right) \cap \operatorname{cl}\left(P_{4}\right)=\left\{\mathbf{x} \in \mathbb{R}^{3}: 2 x_{1}-x_{3}=2 \gamma\right\},
\end{aligned}
$$


which fulfill that

$$
\begin{aligned}
& S W_{i,(i+1)} \cap\left\{\mathbf{x} \in \mathbb{R}^{3}: x_{3}>0\right\} \in P_{i}, \\
& S W_{i,(i+1)} \cap\left\{\mathbf{x} \in \mathbb{R}^{3}: x_{3} \leq 0\right\} \in P_{i+1} .
\end{aligned}
$$

The switching surfaces given in (33) along with the condition given in (34) ensure the existence of the four heteroclinic orbits. Moreover, $\operatorname{cl}\left(W_{\mathbf{x}_{e q_{2}}^{*}}^{u}\right) \cap W_{\mathbf{x}_{e_{q_{3}}}^{*}}^{s} \neq \varnothing$ and $c l\left(W_{\mathbf{x}_{q_{q_{3}}}^{u}}^{u}\right) \cap W_{\mathbf{x}_{q_{2}}^{*}}^{s} \neq \varnothing$.

$\mathrm{As}^{\mathrm{eq}_{3}}$ example, consider the system with parameters $a=0.2, b=5, c=-3, \alpha=1$, and $\gamma=10$, the system presents two self-excited attractors; however, the hidden double-scroll attractor is not present and a transitory double-scroll oscillation is exhibited instead. Figure 2(a) shows in blue the trajectory for the initial condition $\mathbf{x}_{0}=(0,0,0)^{T}$ for a time $t \in[0,150]$ a.u. (arbitrary units). Thus, an enough separation of the self-excited double-scroll attractors is not sufficient to produce a hidden double-scroll attractor.

The absence of a hidden attractor is related to the switching plane $S W_{2,3}$ and how the trajectories of the transitory double-scroll cross it near the stable manifolds. Consider now the following switching surfaces:

$$
\begin{aligned}
& S W_{1,2}=\operatorname{cl}\left(P_{1}\right) \cap \operatorname{cl}\left(P_{2}\right)=\left\{\mathbf{x} \in \mathbb{R}^{3}: 2 x_{1}-x_{3}=-2 \gamma, x_{3}<0\right\}, \\
& S W_{2,3}=\operatorname{cl}\left(P_{2}\right) \cap \operatorname{cl}\left(P_{3}\right)=\left\{\mathbf{x} \in \mathbb{R}^{3}: x_{1}=0\right\}, \\
& S W_{3,4}=\operatorname{cl}\left(P_{3}\right) \cap \operatorname{cl}\left(P_{4}\right)=\left\{\mathbf{x} \in \mathbb{R}^{3}: 2 x_{1}-x_{3}=2 \gamma, x_{3}>0\right\} .
\end{aligned}
$$

With the change in $S W_{2,3}$, the intersections $c l\left(W_{\mathbf{x}_{e q_{2}}^{*}}^{u}\right) \cap W_{\mathbf{x}_{e q_{3}}^{*}}^{s}=\varnothing$ as well as $c l\left(W_{\mathbf{x}_{e q_{3}}^{*}}^{u}\right) \cap W_{\mathbf{x}_{e q_{2}}^{*}}^{s}=\varnothing$. Furthermore, the distance from $\operatorname{cl}\left(W_{\mathbf{x}_{e_{2}}^{*}}^{u}\right) \cap S W_{2,3}$ to $W_{\mathbf{x}_{e q_{3}}^{*}}^{s} \cap S W_{2,3}$ and by symmetry from $c l\left(W_{\mathbf{x}_{e_{2}}^{*}}^{u}\right) \cap S W_{2,3}$ to $W_{\mathbf{x}_{e q}^{*}}^{s} \cap S W_{2,3}$ allow the trajectories to cross $S W_{2,3}$ far from the stable manifolds and allowing the existence of the hidden double-scroll attractor.

As example, consider the system given by (1), (2), (4), and (30) with $a=0.2, b=5, c=-3, \alpha=1$, and $\gamma=10$ and the new switching surfaces given by (35). Figure 2(b) shows in blue the trajectory for the initial condition $\mathbf{x}_{0}=(0,0,0)^{T}$ for a time $t \in[50000,51000]$ a.u. The trajectory reaches the hidden doublescroll instead of converging to one of the self-excited attractors. A trajectory has been simulated for a time $t>1000000$ to verify that the double-scroll oscillation is not a transitory behavior and that the trajectory does not converge to a self-excited attractor.

The construction can be further extended to the number of scrolls desired just by adding two atoms for each scroll; for instance, for a triple scroll attractor the partition is $P=\left\{P_{1}, \ldots\right.$, $\left.P_{6}\right\}$ and a possible set of switching surfaces is given by

$$
\begin{aligned}
& S W_{1,2}=\operatorname{cl}\left(P_{1}\right) \cap \operatorname{cl}\left(P_{2}\right)=\left\{\mathbf{x} \in \mathbb{R}^{3}: 2 x_{1}-x_{3}=-4 \gamma, x_{3}<0\right\}, \\
& S W_{2,3}=\operatorname{cl}\left(P_{2}\right) \cap \operatorname{cl}\left(P_{3}\right)=\left\{\mathbf{x} \in \mathbb{R}^{3}: x_{1}=-\gamma\right\}, \\
& S W_{3,4}=\operatorname{cl}\left(P_{3}\right) \cap \operatorname{cl}\left(P_{4}\right)=\left\{\mathbf{x} \in \mathbb{R}^{3}: 2 x_{1}-x_{3}=0, x_{3}>0\right\}, \\
& S W_{4,5}=\operatorname{cl}\left(P_{4}\right) \cap \operatorname{cl}\left(P_{5}\right)=\left\{\mathbf{x} \in \mathbb{R}^{3}: x_{1}=\gamma\right\}, \\
& S W_{5,6}=\operatorname{cl}\left(P_{5}\right) \cap c l\left(P_{6}\right)=\left\{\mathbf{x} \in \mathbb{R}^{3}: 2 x_{1}-x_{3}=4 \gamma, x_{3}>0\right\},
\end{aligned}
$$

with

$$
f(\mathbf{x})= \begin{cases}-\alpha-2 \gamma, & \mathbf{x} \in P_{1}, \\ \alpha-2 \gamma, & \mathbf{x} \in P_{2}, \\ -\alpha, & \mathbf{x} \in P_{3}, \\ \alpha, & \mathbf{x} \in P_{4}, \\ -\alpha+2 \gamma, & \mathbf{x} \in P_{3}, \\ \alpha+2 \gamma, & \mathbf{x} \in P_{4} .\end{cases}
$$

Then, the equilibria are located at the $x_{1}$-axis with $x_{1 e q_{1}}^{*}=-2 \gamma-\alpha, \quad x_{1 e q_{2}}^{*}=-2 \gamma+\alpha, \quad x_{1 e q_{3}}^{*}=-\alpha, \quad x_{1 e q_{4}}^{*}=\alpha$, $x_{1 e q_{5}}^{*}=2 \gamma-\alpha$, and $x_{1 e q_{6}}^{*}=2 \gamma+\alpha$.

\section{Generalization}

The number of scrolls for a hidden scroll attractor exhibited by the system from the previous section depends on the number of self-excited attractors. In order to simplify the description for a large number of scrolls, a generalization is introduced in this section. Consider a dynamical system $T: X \longrightarrow X$ whose dynamic is given by

$$
\dot{x}=A \mathbf{x}+B F(\mathbf{x}),
$$

where $\mathbf{x}=\left(x_{1}, x_{2}, x_{3}\right)^{T} \in \mathbb{R}^{3}$ is the state vector, $A=\left\{\alpha_{i j}\right\} \in \mathbb{R}^{3 \times 3}$ is the linear operator given by (2), and $F$ is a functional such that $B F(\mathbf{x})$ is a constant vector in each atom $P_{i}$. The saddle equilibrium point of each atom is given by $\mathbf{x}_{e q_{i}}^{*}=\left(x_{1_{e q_{i}}}^{*}, x_{2_{e q_{i}}}^{*}, x_{3_{e q q_{i}}}^{*}\right)^{T}=(F(\mathbf{x}), 0,0)^{T}$, with $i=1, \ldots, \eta$. $F(\mathbf{x})$ is defined as follows:

$$
F(\mathbf{x})=\alpha g\left(2\left(x_{1}-f_{1}\left(x_{1}\right)\right)-x_{3}, x_{3}\right)+f_{1}\left(x_{1}\right),
$$

where

$$
f_{1}\left(x_{1}\right)=\sum_{j=1}^{N_{x_{1}}} \gamma u\left(x_{1}+2 \gamma(j-1)-\gamma\left(N_{x_{1}}-1\right)\right),
$$

with $N_{x_{1}} \in \mathbb{Z}^{+}$. The parameters $\alpha$ and $\gamma$ fulfill Assumption 3 . The function $u(y)$ is the Heaviside step function:

$$
u(y)= \begin{cases}1, & \text { if } y \geq 0, \\ -1, & \text { if } y<0,\end{cases}
$$

and $g$ is a step function defined as follows:

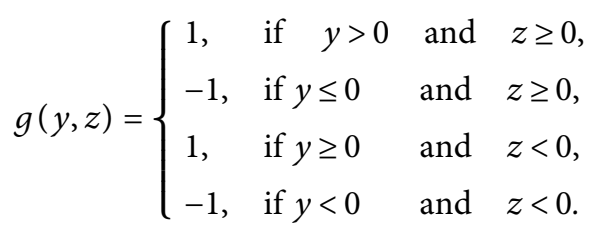

Note that $g(y, z)$ is equal to $u(y)$, when $z<0$; while, for $z \geq 0$, it is similar to $u(y)$ with the only difference that 0 is mapped to 1 instead of -1 .

Please note that $-B$ is the first column of the operator $A$ and therefore system (38) can be rewritten as follows:

$$
\dot{x}=A \mathbf{x}-A B(\mathbf{x}),
$$




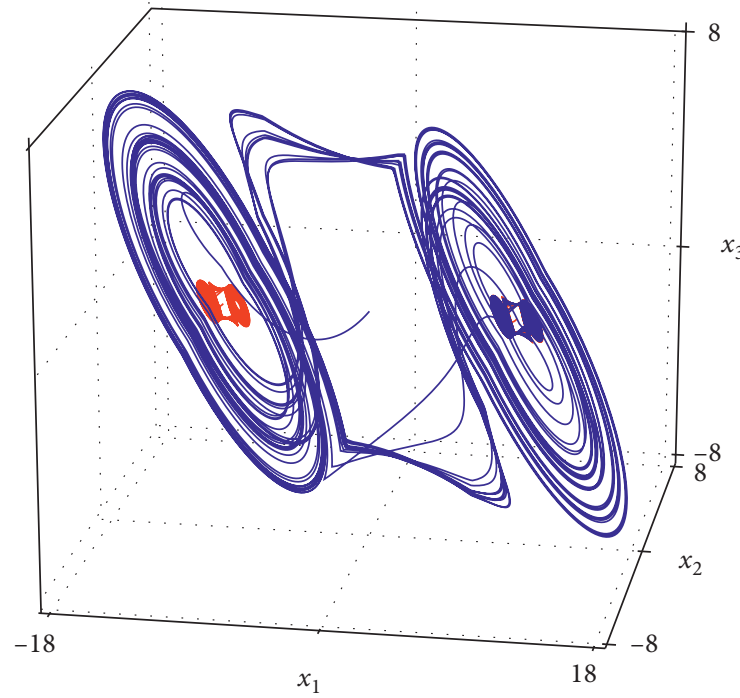

(a)

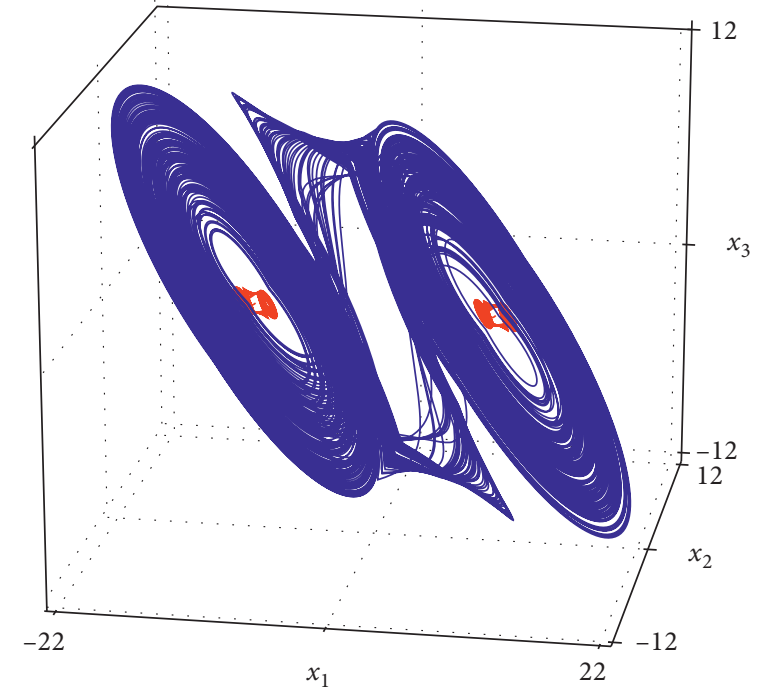

(b)

Figure 2: Simulation of the system given by (1), (2), (4), and (30) with $a=0.2, b=5, c=-3, \alpha=1, \gamma=10$, and $\alpha=1$ for the initial condition $x=(0,0,0)^{T}$ in blue. In (a) the surfaces are defined by (33) and (34), the simulation is shown for $t \in[0,300] a \cdot u$. (arbitrary units), and the trajectory converges to one of the self-excited attractors in red. In (b) the surfaces are defined by (35) and the simulation is shown for $t \in[50000,51000] a \cdot u$., and the hidden attractor emerges.

where $B: X \longrightarrow X$ is a vector valued function defined as follows:

$$
B(\mathbf{x})=\left(\begin{array}{c}
B_{1} \\
B_{2} \\
B_{3}
\end{array}\right)=\left(\begin{array}{c}
\alpha g\left(2\left(x_{1}-f_{1}\left(x_{1}\right)\right)-x_{3}, x_{3}\right)+f_{1}\left(x_{1}\right) \\
0 \\
0
\end{array}\right),
$$

such that $A B(x)$ is a constant vector in each atom $P_{i}$. The saddle equilibrium point of each atom is now given by $\mathbf{x}_{e q_{i}}^{*}=\left(x_{1_{e q}}^{*}, x_{2_{e q}}^{*}, x_{3 e q}^{*}\right)^{T}=B(\mathbf{x})$, with $i=1, \ldots, \eta$.

To understand the form of $B(\mathbf{x})$, it is useful to separate $B_{1}$ and analyze the effect of each term of the sum, i.e., the effect of the term $\alpha g\left(2\left(x_{1}-f_{1}\left(x_{1}\right)\right)-x_{3}, x_{3}\right)$ and the effect of $f_{1}\left(x_{1}\right)$.

First, consider the function $f_{1}\left(x_{1}\right)$ whose plot resembles a stair centered at the origin whose plateaus are of $2 \gamma$ in height and width. Two examples for $N_{x_{1}}=3$ and $N_{x_{1}}=6$ are shown in Figure 3.

Thus, $f_{1}\left(x_{1}\right)$ generates $N_{x_{1}}$ switching planes of the form $\left\{\mathbf{x} \in \mathbb{R}^{3}: x_{1}=\epsilon \in \mathbb{R}\right\}$, which are parallel to the plane $x_{2}-x_{3}$. Then, $f_{1}\left(x_{1}\right)$ generates a partition $R=\left\{R_{1}, \ldots, R_{N_{x_{1}}+1}\right\}$ of $X$.

Now, consider the term $\alpha g\left(2\left(x_{1}-f_{1}\left(x_{1}\right)\right)-x_{3}, x_{3}\right)$, and this term generates a switching plane $\left\{\mathbf{x} \in R_{i}: 2\left(x_{1}-\right.\right.$ $\left.\left.f_{1}\left(x_{1}\right)\right)-x_{3}=0\right\}$ for $i=1, \ldots, N_{x}+1$. Since $f_{1}\left(x_{1}\right)$ takes $N_{x_{1}}+1$ values, then $\alpha g\left(2\left(x_{1}-f_{1}\left(x_{1}\right)\right)-x_{3}, x_{3}\right)$ generates $N_{x_{1}}+1$ switching planes, one for each element of the partition $R$. Thus, the elements $R_{i}$ are split and the partition $P=\left\{P_{1}, \ldots, P_{2 N_{x_{1}}+2}\right\}$ is generated.

In this way, $B(\mathbf{x})$ locates two equilibrium points with a separation of $2 \alpha$ in the middle of each element $R_{i}$ (along $x_{1}$ ) for $i=1, \ldots, N_{x_{1}}+1$. In the partition $P, B(\mathbf{x})$ locates an equilibrium point in each $P_{i}$ for $i=1, \ldots, 2 N_{x_{1}}+2$.
Thus, the equilibria along the $x_{1}$-axis is located in $N_{x_{1}}$ pairs, each pair of nearby equilibrium points have a separation of $2 \alpha$. Let us denote the midpoint of the line that joins a pair $\mu$ of nearby equilibria as $c p_{\mu}$ with $\mu=1, \ldots,\left(N_{x_{1}}+1\right)$. Then, the distance from $c p_{i}$ to $c p_{i+1}$ is $2 \gamma$. The purpose of this distribution for the equilibria is to allow the existence of double-scroll self-excited attractors that are separated enough from other double-scroll self-excited attractors in a way that these resemble equilibria for the generation of a bigger scroll attractor at a larger scale. This larger scroll attractor is indeed the hidden scroll attractor.

The equilibria are located along the $x_{1}$-axis as follows:

$$
\begin{aligned}
x_{1 e q_{1}}^{*} & =(0) 2 \gamma-\gamma\left(N_{x_{1}}\right)-\alpha, \\
x_{1 e q_{3}}^{*} & =(1) 2 \gamma-\gamma\left(N_{x_{1}}\right)-\alpha, \\
x_{1 e q_{5}}^{*} & =(2) 2 \gamma-\gamma\left(N_{x_{1}}\right)-\alpha, \\
\vdots & \\
x_{1 e q_{2 N_{x_{1}-1}}}^{*} & =\left(N_{x_{1}}-1\right) 2 \gamma-\gamma\left(N_{x_{1}}\right)-\alpha, \\
x_{1 e q_{2 N_{x_{1}+1}}}^{*} & =\left(N_{x_{1}}\right) 2 \gamma-\gamma\left(N_{x_{1}}\right)-\alpha, \\
x_{1 e q_{2}}^{*} & =(0) 2 \gamma-\gamma\left(N_{x_{1}}\right)+\alpha, \\
x_{1 e q_{4}}^{*} & =(1) 2 \gamma-\gamma\left(N_{x_{1}}\right)+\alpha, \\
x_{1 e q_{6}}^{*} & =(2) 2 \gamma-\gamma\left(N_{x_{1}}\right)+\alpha, \\
\vdots & \\
x_{1 e q_{2 N_{x_{1}}}}^{*} & =\left(N_{x_{1}}-1\right) 2 \gamma-\gamma\left(N_{x_{1}}\right)+\alpha, \\
x_{1 e q_{2 N_{x_{1}}+2}}^{*} & =\left(N_{x_{1}}\right) 2 \gamma-\gamma\left(N_{x_{1}}\right)+\alpha .
\end{aligned}
$$




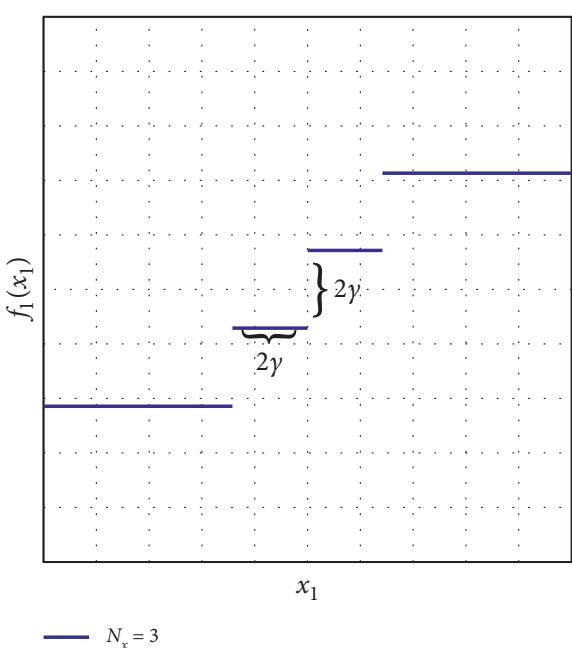

(a)

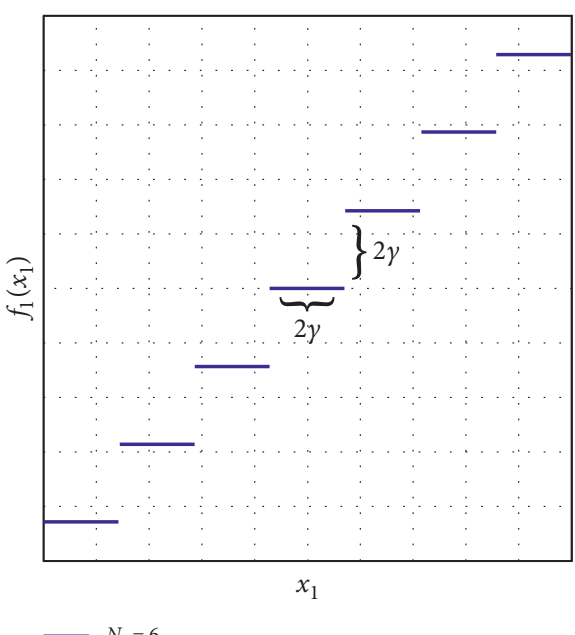

(b)

Figure 3: The plot of the function $f_{1}\left(x_{1}\right)$ for (a) $N_{x_{1}}=3$ and (b) $N_{x_{1}}=6$.

The switching planes located in the middle of the selfexcited attractors are given as follows:

$$
\begin{aligned}
S W_{1,2}=\left\{\mathbf{x} \in \mathbb{R}^{3}: 2\left(x_{1}-\left(x_{1 e q_{1}}^{*}+\alpha\right)\right)-x_{3}=0,\right. & \left.x_{1}<x_{1 e q_{2}}^{*}+(\gamma-\alpha)\right\}, \\
S W_{3,4}= & \left\{\mathbf{x} \in \mathbb{R}^{3}: 2\left(x_{1}-\left(x_{1 e q_{3}}^{*}+\alpha\right)\right)-x_{3}=0, \quad x_{1 e q_{3}}^{*}-(\gamma-\alpha) \leq x_{1}<x_{1 e q_{4}}^{*}+(\gamma-\alpha)\right\}, \\
\vdots & \\
S W_{2 N_{x_{1}}+1,2 N_{x_{1}}+2}=\left\{\mathbf{x} \in \mathbb{R}^{3}: 2\left(x_{1}-\left(x_{1 e q_{N_{x_{1}}+1}}^{*}+\alpha\right)\right)-x_{3}=0,\right. & \left.x_{e q_{2 N_{x_{1}}+1}}^{*}-(\gamma-\alpha) \leq x_{1}\right\} .
\end{aligned}
$$

The rest of the switching surfaces are

$$
\begin{aligned}
S W_{2,3} & =\left\{\mathbf{x} \in \mathbb{R}^{3}: x=x_{1 e q_{2}}^{*}+(\gamma-\alpha)\right\}, \\
S W_{4,5} & =\left\{\mathbf{x} \in \mathbb{R}^{3}: x=x_{1 e q_{4}}^{*}+(\gamma-\alpha)\right\}, \\
& \vdots \\
S W_{2 N_{x_{1}}-2,2 N_{x_{1}}-1} & =\left\{\mathbf{x} \in \mathbb{R}^{3}: x=x_{1 e q_{2 N_{x_{1}-2}}}^{*}+(\gamma-\alpha)\right\}, \\
S W_{2 N_{x_{1}}, 2 N_{x_{1}}+1} & =\left\{\mathbf{x} \in \mathbb{R}^{3}: x=x_{1 e q_{2 N_{x_{1}}}}^{*}+(\gamma-\alpha)\right\} .
\end{aligned}
$$

To illustrate the construction, consider the parameters $a=0.2, b=5, c=-7$, and $N_{x_{1}}=1$, the system presents two self-excited attractors and a hidden double-scroll attractor, which is shown in Figure 4(a).

According to the definition, the attraction basin of a hidden attractor does not intersect neighborhoods of equilibria. Figure 5 shows the cut of the numerically evaluated basins of attraction given by the plane $x_{3}=0$. Each double-scroll self-excited attractor has its own attraction basin shown in red and green. Also, the attractor around these double-scroll self-excited attractors has its own attraction basin shown in blue and the intersection of this basin with the attraction basins of the self-excited attractor is the empty set. Because all equilibria of the system belong to the attraction basin of the self-excited attractors, the attraction basin of the attractor around the self-excited attractors does not contain an equilibrium point. So, the attractor around the self-excited attractors is a hidden attractor.

Another numerical approach to verify that it is a hidden attractor consist in performing a long-time simulation of a trajectory and make sure that the trajectory does not converge to a self-excited attractor. In Figure 6, it is shown that the simulation for the initial condition $\mathbf{x}_{0}=(0,0,0)^{T}$ with $t \in[1000000,10001000]$. This last approach requires less computing time, so it was the approach used in all the examples in the manuscript.

For a second example, consider $N_{x_{1}}=4$; then, the system presents five self-excited attractors and a hidden 5-scroll attractor shown in Figure 4(b). Thus, the number of scrolls is equal to the number of self-excited attractors, which is $N_{x_{1}}+1$.

\section{Extension for 2D and 3D Grid Scroll Hidden Attractors}

The approach presented in Section 4 can be further extended for $2 \mathrm{D}$ and $3 \mathrm{D}$ grid scroll hidden attractors. The idea is to 


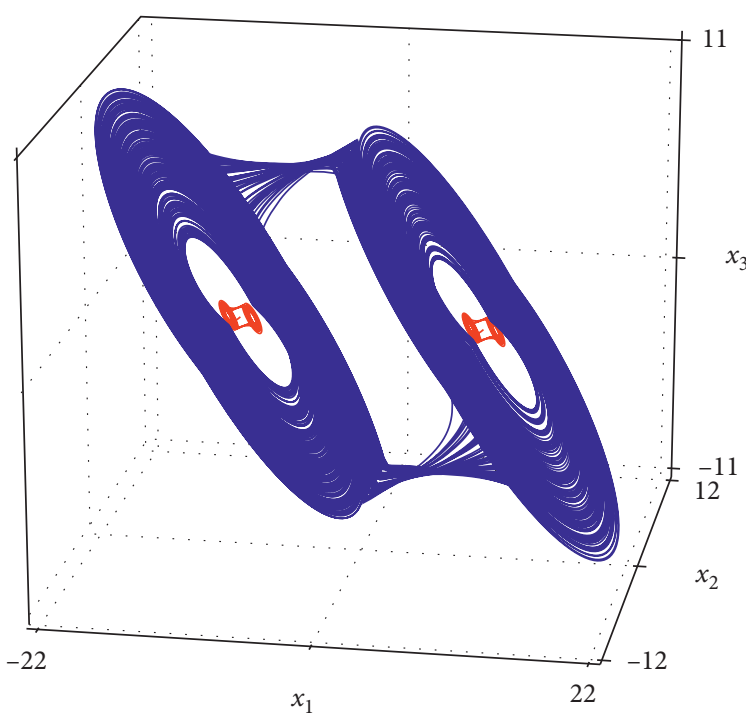

(a)

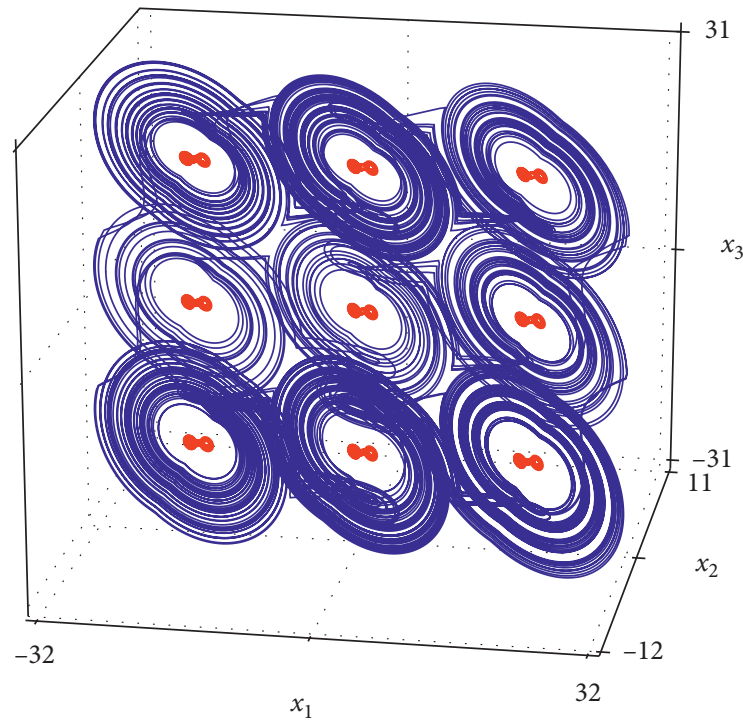

(c)

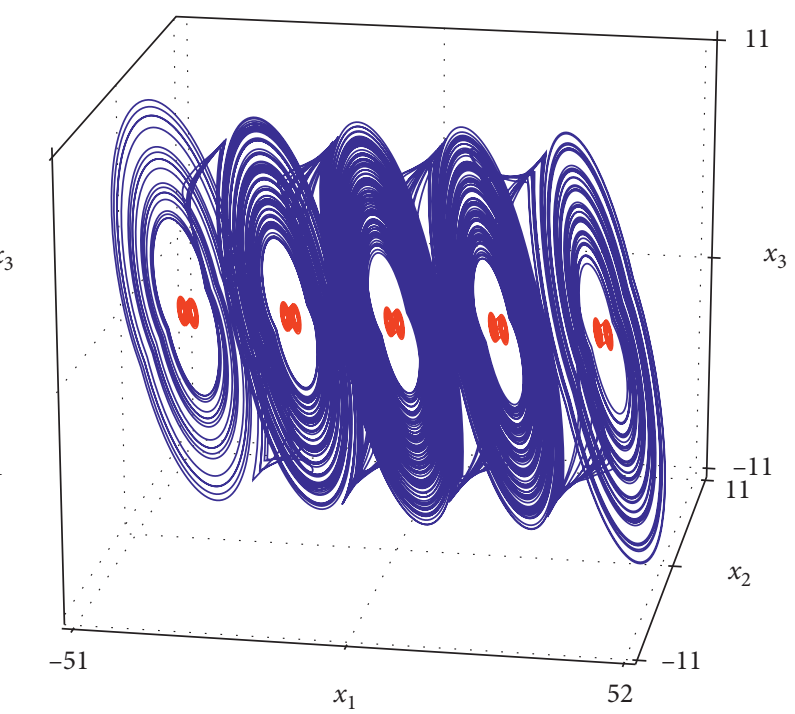

(b)

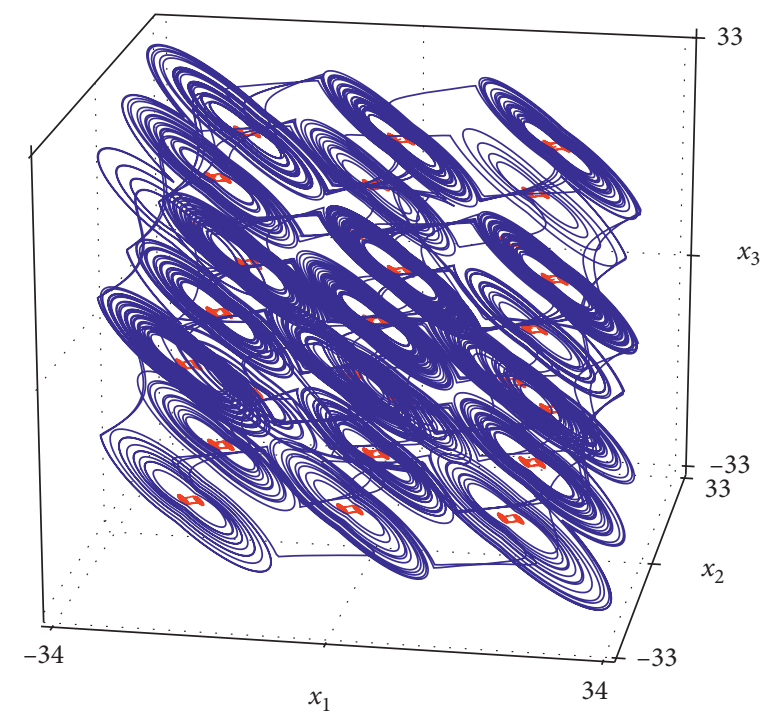

(d)

FIgURE 4: Simulation of the hidden attractor (in blue) and the self-excited attractors (in red) exhibited by system (43) with parameters $a=0.2, b=5$, and $c=-7$. In (a) and (b) $B(\mathbf{x})$ is given by (44) with $N_{x_{1}}=1$ and $N_{x_{1}}=4$, respectively. In (c) $B(\mathbf{x})$ is given by (48) with $E_{2}=0, E_{3}=1, N_{x_{1}}=2$, and $N_{x_{3}}=2$. In (d) $B(\mathbf{x})$ is given by (48) with $E_{2}=1, E_{3}=1, N_{x_{1}}=2, N_{x_{2}}=2, N_{x_{3}}=2$, and $w=0.2$.

add switching surfaces, which are parallel to the planes $x_{1}-$ $x_{2}$ and $x_{1}-x_{3}$.

This requires the modification on $B(\mathbf{x})$, for simplicity, the functions $f_{i}(\cdot)$ are written as $f_{i}$ :

$$
B(\mathbf{x})=\left(\begin{array}{c}
f_{4}+f_{1}-\frac{w f_{2}}{\gamma} \\
f_{2} \\
f_{3}+\frac{w f_{2}}{\gamma}
\end{array}\right),
$$

$$
f_{2}=E_{2}\left(\sum_{k=1}^{N_{x_{2}}} \gamma u\left(x_{2}+2 \gamma(k-1)-\gamma\left(N_{x_{2}}-1\right)\right)\right),
$$

$$
f_{3}=E_{3}\left(\sum_{l=1}^{N_{x_{3}}} \gamma u\left(\left(x_{3}-\frac{w f_{2}}{\gamma}\right)+2 \gamma(l-1)-\gamma\left(N_{x_{3}}-1\right)\right)\right),
$$

$$
f_{1}=\sum_{j=1}^{N_{x_{1}}} \gamma u\left(\left(x_{1}+\frac{w f_{2}}{\gamma}\right)+2 \gamma(j-1)-\gamma\left(N_{x_{1}}-1\right)\right),
$$

where 


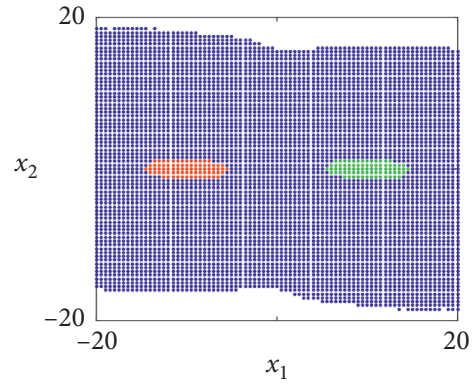

(a)

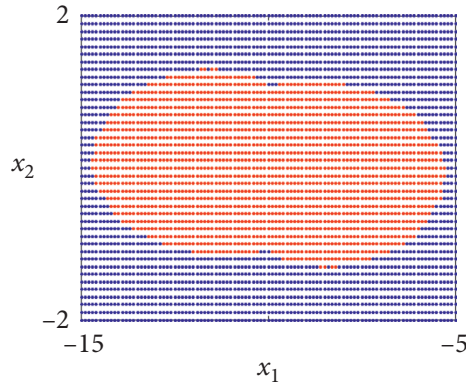

(b)

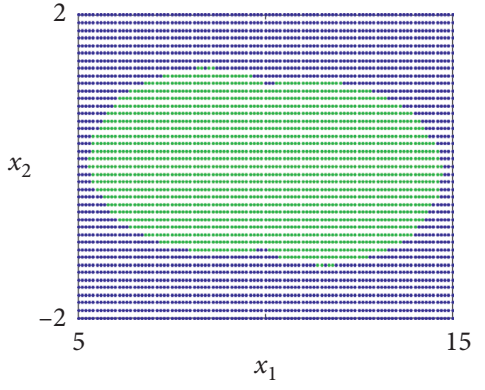

(c)

Figure 5: Cut of basins of attraction of system (43) with parameters $a=0.2, b=5, c=-7$, and $N_{x_{1}}=1$ at $x_{3}=0$. Red and green dots belong to the basins of attraction of self-excited attractors, and blue dots belong to the basin of attraction of the hidden attractor. In (a) grid of 0.5 and in (b) and (c) grid of 0.1 .

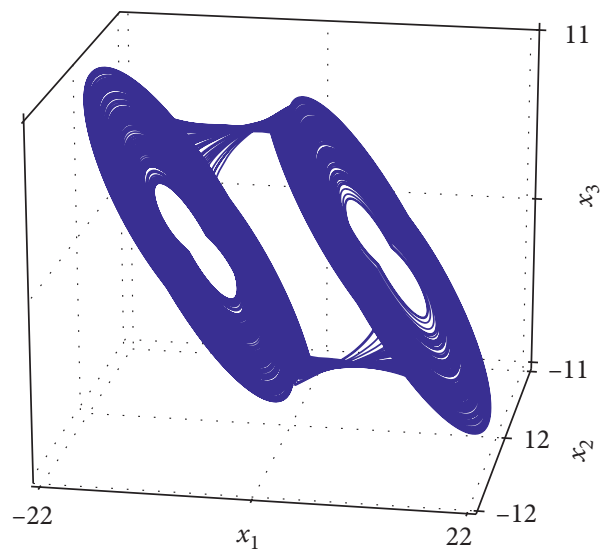

FiguRE 6: Simulation of the hidden attractor exhibited by system (43) with parameters $a=0.2, b=5, c=-7$, and $N_{x_{1}}=1$ for $t \in[1000000,10001000]$.

$$
\begin{aligned}
f_{4}=\alpha g\left(2\left(x_{1}+\frac{w f_{2}}{\gamma}-f_{1}\right)\right. & -\left(x_{3}-\frac{w f_{2}}{\gamma}-f_{3}\right), \\
& \left.\left(x_{3}-\frac{w f_{2}}{\gamma}-f_{3}\right)\right),
\end{aligned}
$$

where (52) $w \geq 0, E_{2}, E_{3} \in\{0,1\}$, and $N_{x_{1}}, N_{x_{2}}, N_{x_{3}} \in \mathbb{Z}^{+}$.

Let us denote the equilibria found in Section 4 as $\mathbf{x}_{e q_{j}}^{* 1 D}=$ $\left(x_{1 e q_{j}}^{* 1 D}, x_{2 e q_{j}}^{* 1 D}, x_{3 e q_{j}}^{* 1 D}\right)^{T}$ for $j=1, \ldots, 2 N_{x_{1}}+2$. Then, the new equilibria for the new $B(\mathbf{x})$ in (48) are found from

$$
\mathbf{x}_{j k l}^{* 3 D}=\mathbf{x}_{e q_{j}}^{* 1 D}+\left(\begin{array}{c}
0 \\
0 \\
\left(E_{1} l\right) 2 \gamma-\gamma N_{x_{3}}
\end{array}\right)+\left(\begin{array}{c}
0 \\
\left(E_{2} k\right) 2 \gamma-\gamma N_{x_{2}} \\
0
\end{array}\right)+\left(\begin{array}{c}
-\frac{w f_{2}(y)}{\gamma} \\
0 \\
\frac{w f_{2}(y)}{\gamma}
\end{array}\right),
$$

where $k=1, \ldots, 2 N_{x_{2}}+2$ and $l=1, \ldots, 2 N_{x_{3}}+2$. The switching surfaces are now restricted on $x_{2}$ and $x_{3}$ and located according to the new equilibria.

As example, consider the parameters $a=0.2, b=5$, $c=-7$, and $w=0.2$ with $E_{2}=0, E_{3}=1, N_{x_{1}}=2$, and $N_{x_{3}}=$ 2 for a 2D-grid scroll hidden attractor shown in Figure 4(c) and $E_{2}=1, E_{3}=1, N_{x_{1}}=2, N_{x_{3}}=2$, and $N_{x_{3}}=2$ for a $3 \mathrm{D}$ grid scroll hidden attractor shown in Figure $4(\mathrm{~d})$.

\section{Conclusions}

In this work, the question of whether or not it is possible to generate a hidden multiscroll attractor with an arrangement of scrolls along more than one direction from multiple selfexcited attractors was addressed. It was found that the separation between self-excited double-scroll attractors and the switching plane between these self-exited attractors lead 
to the emergence of a hidden attractor. A generalized construction was proposed for the generation of multistable systems with self-excited double-scroll chaotic attractors and a hidden multiscroll/grid attractor. The coexistence of selfexcited attractors and a hidden attractor is presented via PWL systems and the approach considers for each scroll in the hidden attractors a self-excited attractor inside the scroll. As future work, we envision working on the answer to the following question: is it possible to generate multistability with more than one hidden attractor?

\section{Data Availability}

The data used to support the findings of this study are included within the article.

\section{Conflicts of Interest}

The authors declare that there are no conflicts of interest regarding the publication of this paper.

\section{Acknowledgments}

R.J. Escalante-González is thankful to CONACYT for the scholarships granted. Eric Campos acknowledges CONACYT for the financial support through Project no. A1-S30433.

\section{References}

[1] E. Campos-Cantón, J. G. Barajas-Ramírez, G. Solís-Perales, and R. Femat, "Multiscroll attractors by switching systems. Chaos," An Interdisciplinary Journal of Nonlinear Science, vol. 20, no. 1, 2010.

[2] L. J. Ontañón-García and E. Campos-Cantón, "Widening of the basins of attraction of a multistable switching dynamical system with the location of symmetric equilibria," Nonlinear Analysis: Hybrid Systems, vol. 26, pp. 38-47, 2017.

[3] G. A. Leonov, N. V. Kuznetsov, and V. I. Vagaitsev, "Localization of hidden Chua's attractors," Physics Letters A, vol. 375 , no. 23 , pp. $2230-2233,2011$.

[4] D. Dudkowski, S. Jafari, T. Kapitaniak, N. V. Kuznetsov, G. A. Leonov, and A. Prasad, "Hidden attractors in dynamical systems," Physics Reports, vol. 637, no. 1, p. 50, 2016.

[5] E. N. Lorenz, "Deterministic nonperiodic flow," Joutnal of the Atmospheric Sciences, vol. 20, pp. 130-141, 1963.

[6] T. Matsumoto, "A chaotic attractor from Chua's circuit," IEEE Transactions on Circuits and Systems, vol. 31, no. 12, pp. 1055-1058, 1984.

[7] S. Jafari, J. C. Sprott, and S. M. R. Hashemi Golpayegani, "Elementary quadratic chaotic flows with no equilibria," Physics Letters A, vol. 377, no. 9, pp. 699-702, 2013.

[8] D. Cafagna and G. Grassi, "Chaos in a new fractional-order system without equilibrium points," Communications in Nonlinear Science and Numerical Simulation, vol. 19, no. 9, pp. 2919-2927, 2014.

[9] Z. Wang, S. Cang, E. O. Ochola, and Y. Sun, "A hyperchaotic system without equilibrium," Nonlinear Dynamics, vol. 69, no. 1-2, pp. 531-537, 2012.

[10] C. Li, J. C. Sprott, W. Thio, and H. Zhu, "A new piecewise linear hyperchaotic circuit," IEEE Transactions on Circuits and Systems II: Express Briefs, vol. 61, no. 12, pp. 977-981, 2014.

[11] J. M. Munoz-Pacheco, E. Zambrano-Serrano, C. Volos, S. Jafari, J. Kengne, and K. Rajagopal, “A new fractional-order chaotic system with different families of hidden and selfexcited attractors," Entropy, vol. 20, no. 8, 2018.

[12] X. Hu, C. Liu, L. Liu, J. Ni, and S. Li, "Multi-scroll hidden attractors in improved Sprott A system," Nonlinear Dynamics, vol. 86, no. 3, pp. 1725-1734, 2016.

[13] X. Hu, C. Liu, L. Liu, Y. Yao, and G. Zheng, "Multi-scroll hidden attractors and multi-wing hidden attractors in a 5dimensional memristive system," Chinese Physics B, vol. 26, no. 11, p. 110502, 2017.

[14] F. R. Tahir, S. Jafari, V.-T. Pham, C. Volos, and X. Wang, "A novel no-equilibrium chaotic system with multiwing butterfly attractors," International Journal of Bifurcation and Chaos, vol. 25, no. 04, p. 1550056, 2015.

[15] M. A. Kiseleva, N. V. Kuznetsov, and G. A. Leonov, "Hidden attractors in electromechanical systems with and without equilibria," IFAC-PapersOnLine, vol. 49, no. 14, pp. 51-55, 2016.

[16] M.-F. Danca, N. Kuznetsov, and G. Chen, "Unusual dynamics and hidden attractors of the Rabinovich-Fabrikant system," Nonlinear Dynamics, vol. 88, no. 1, pp. 791-805, 2017.

[17] R. J. Escalante-González and E. Campos-Cantón, "Generation of chaotic attractors without equilibria via piecewise linear systems," International Journal of Modern Physics C, vol. 28, no. 1, 2017.

[18] R. J. Escalante-González, E. Campos-Cantón, and M. Nicol, "Generation of multi-scroll attractors without equilibria via piecewise linear systems," Chaos: An Interdisciplinary Journal of Nonlinear Science, vol. 27, no. 5, 2017.

[19] R. J. Escalante-Gonzalez and E. Campos-Canton, "A class of piecewise linear systems without equilibria with 3-d grid multiscroll chaotic attractors," IEEE Transactions on Circuits and Systems II: Express Briefs, vol. 66, no. 8, pp. 1456-1460, 2019.

[20] J. Ma, F. Wu, W. Jin, P. Zhou, and T. Hayat, "Calculation of Hamilton energy and control of dynamical systems with different types of attractors," Chaos: An Interdisciplinary Journal of Nonlinear Science, vol. 27, no. 5, 2017.

[21] G. Zhang, F. Wu, C. Wang, and J. Ma, "Synchronization behaviors of coupled systems composed of hidden attractors," International Journal of Modern Physics B, vol. 31, no. 26, p. 1750180, 2017.

[22] J. A. K. Suykens, A. Huang, and L. O. Chua, "A family of n-scroll attractors from a generalized Chua's circuit," Archiv Fur Elektronik und Ubertragungstechnik, vol. 51, no. 3, pp. 131-138, 1997.

[23] W. K. S. Tang, G. Q. Zhong, G. Chen, and K. F. Man, "Generation of N-Scroll attractors via sine function," IEEE Transactions on Circuits and Systems I: Fundamental Theory and Applications, vol. 48, no. 11, pp. 1369-1372, 2001.

[24] M. E. Yalçin, J. A. K. Suykens, J. Vandewalle, and S. Özoğuz, "Families of scroll grid attractors," International Journal of Bifurcation and Chaos, vol. 12, no. 1, pp. 23-41, 2002.

[25] B. Aguirre-Hernández, E. Campos-Cantón, J. A. LópezRenteria, and E. C. Díaz González, "A polynomial approach for generating a monoparametric family of chaotic attractors via switched linear systems," Chaos, Solitons \& Fractals, vol. 71, no. 1, pp. 100-106, 2015.

[26] E. Campos-Cantón, "Chaotic attractors based on unstable dissipative systems via third-order differential equation," International Journal of Modern Physics C, vol. 27, no. 1, 2016. 
[27] S. Jafari, V.-T. Pham, and T. Kapitaniak, "Multiscroll chaotic sea obtained from a simple $3 \mathrm{~d}$ system without equilibrium," International Journal of Bifurcation and Chaos, vol. 26, no. 2, 2016.

[28] R. Sevilla-Escoboza, A. N. Pisarchik, R. Jaimes-Reátegui, and G. Huerta-Cuellar, "Selective monostability in multi-stable systems," Proceedings of the Royal Society A: Mathematical, Physical and Engineering Sciences, vol. 471, 2015.

[29] E. Campos-cantón, R. Femat, J. G. Barajas-ramírez, and I. Campos-cantón, "A multivibrator circuit based on chaos generation," International Journal of Bifurcation and Chaos, vol. 22, no. 1, 2012.

[30] A. Sambas, S. Vaidyanathan, E. Tlelo-Cuautle et al., "A novel chaotic system with two circles of equilibrium points: multistability, electronic circuit and fpga realization," Electronics, vol. 8, no. 11, 2019.

[31] A. Sambas, S. Vaidyanathan, S. Zhang, M. Mamat, and M. A. Mohamed, "Multistability in a novel chaotic system with perpendicular lines of equilibrium: analysis, adaptive synchronization and circuit design," Engineering Letters, vol. 27, 2019.

[32] R. J. Escalante-González and E. Campos-Cantón, "Coexistence of hidden attractors and self-excited attractors through breaking heteroclinic-like orbits of switched systems," Engineering Letters, vol. 27, 2019. 